\title{
Numerical simulation of combustion instabilities under the alternating gravity conditions
}

\author{
Anastasia Krikunova* \\ Moscow Institute of Physics and Technology (State University), 141701, Dolgoprudny, Moscow \\ Region, Russian Federation
}

\begin{abstract}
The work is devoted to the analysis of the methane-air conical flame behaviour under conditions of an alternating gravitational field. Numerical simulation based on the software package FlowVision, has shown the possibility of modeling the flame front instabilities during the transition from the normal gravitational conditions to zero gravity. The appearance of the flame front oscillations is demonstrated under the such conditions. Further studies will provide a complete picture of the behavior of the flame in an alternating gravitational field.
\end{abstract}

\section{Introduction}

Combustion processes experimental studies, both under the weightlessness conditions and under the increased gravity (overload) in centrifuges, Drop towers, ISS and parabolic flights, were carried out by various scientific centers, such as NASA, ESA, Roskosmos [1].In particular, the author of the current work carried out experiments on the vacuumdynamic Bremen Drop tower facility [2 - 4].However, in the literature there is no data on the flame characteristics under transitional conditions (transition from the normal gravity to the microgravity and reverse), which is undoubtedly an essential issue. During the aircraft operating in the modes of such transitions, instabilities may occur, leading to the flame extinction/quenching, which may be critical for the engines stable operation. So the work is of interest, both from the point of view of fundamental research, and for applied activity.

\section{Numericali simulation details}

Numerical simulation of processes by the finite element method was carried out by using a FlowVision software package [5]. A detailed illustration of the nozzle geometry and the computational domain design is shown in the Figure. 1. The burner geometry was identical to the experimental conditions, that were carried out earlier [3]. A conical nozzle was used as the burner, providing a uniform flow of velocity at the outlet section. The geometric dimensions are indicated in Figure 1. Also the flame was stabilized by a ring, placed near the outlet edge of the nozzle. The numerical domain was chosen sufficiently large to avoid

\footnotetext{
* Corresponding author: utro-2007@mail.ru
} 
the influence of the walls on the processes under the investigation, for example the interface between combustion products and ambient area should be free from the far field compression effects. A cylindrical domain with height of $175 \mathrm{~mm}$ and diameter of $70 \mathrm{~mm}$ was used. Normal temperature and pressure were used as the initial conditions; mass flow rate and fuel-oxygen ratio were settled in the inlet boundary condition. From the very beginning the computational domain was filled with air under the normal conditions. The non-uniform 3D grid model was used, it was built from the uniform grid with size $25 \times 19 \times 19$ cells by using 6 order approximation in the vicinity of walls, ring-stabilization body and the flame front region. Finally grid was consisted of amount above $3.3 \mathrm{mln}$ cells. A complete system of Navier-Stokes equations for a viscous compressible gas was simulated. The equations of state of the components corresponded to the model of an ideal gas. The k- $\omega$ SST turbulence model was chosen. To solve the system of linear algebraic equations, an implicit numerical scheme of the second order of accuracy without constraints was used. At the initial stage a time step corresponding to the Courant number of 100 was established to form a developed flow, and then it was decreased to CFL $=20$. Ignition was carried out by raising the temperature at the nozzle edge vicinity for a short time up to $2000^{\circ} \mathrm{K}$, while the CFL was increased to 50 , after the formation of the flow of the combustible mixture. After the formation of a stable flame, the temperature source was removed and the time step was successively reduced up to $10^{-4} \mathrm{~s}$. One-step global reaction model of methane combustion was used to simplify the modeling process and reduce the simulation time. The future work will be devoted to the detailed kinetic scheme introduction into the simulation code.

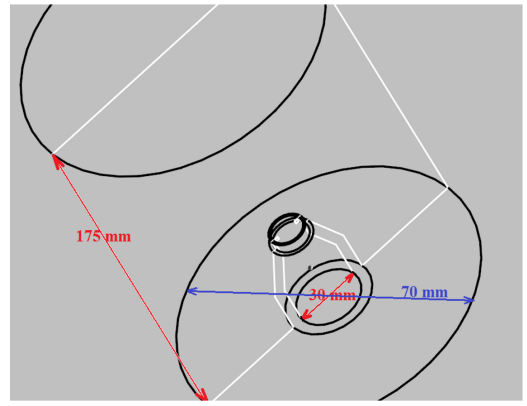

a

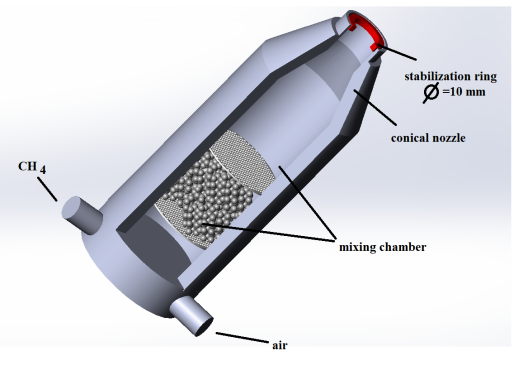

b

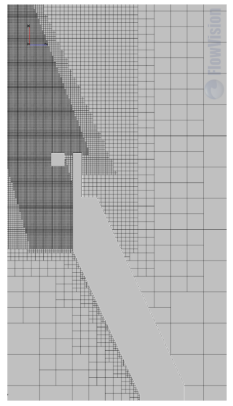

C

Fig. 1. Computational domain (a), burner (b), numerical domain (c).

\section{Results and discussions}

Figure 2 shows the distribution of temperatures and rates of combustion reactions ([kg m-3 s-1]) for normal gravity and zero gravity conditions (transition region). The position of the flame front can be determined from the position of the maximum temperature for the terrestrial gravity (Figures 2a, 2c, 2e), whereas under the transition to weightlessness, the temperature field is redistributed (Figures $2 \mathrm{~b}, 2 \mathrm{~d}, 2 \mathrm{f}$ ). The position of the maximum temperatures region does not correspond to the region of maximum reaction rates and the position of the combustion flame front, since under reduced gravity conditions there are no convective flows that play a major role. 


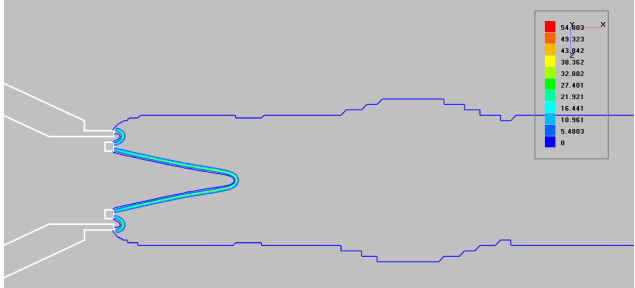

a

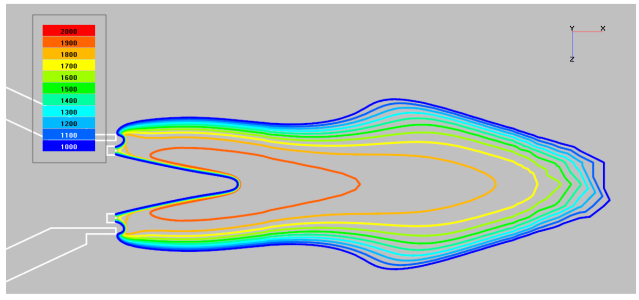

C

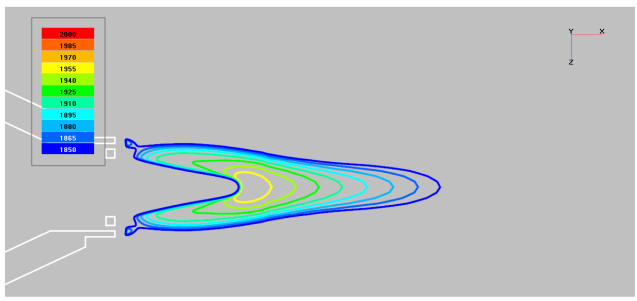

e
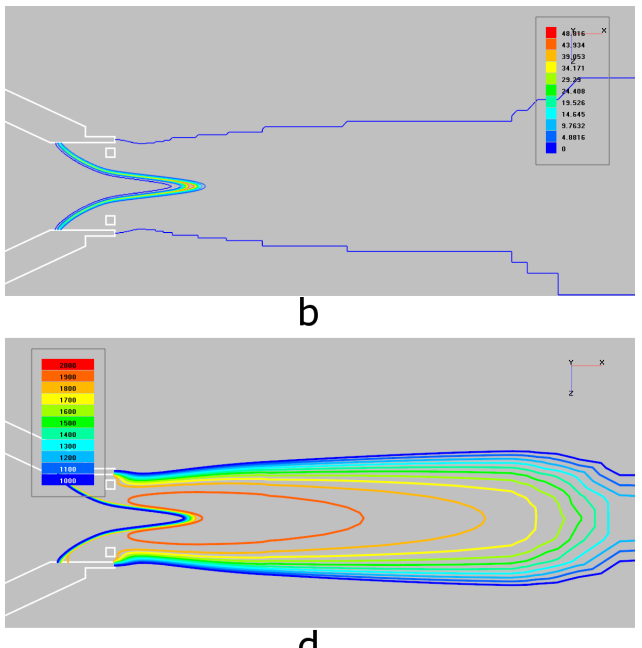

d

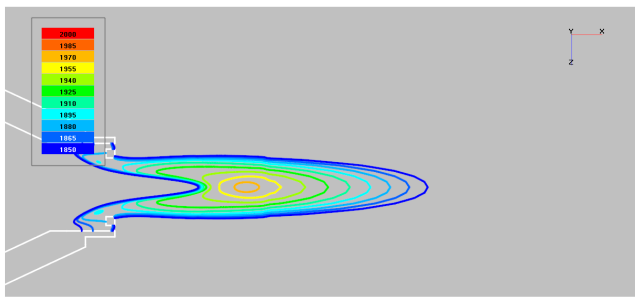

$f$

Fig. 2. Rates of combustion reactions under normal gravity conditions (a) and under the transition to the microgravity (b), temperature distribution for the normal gravity (c), (e) and transition conditions (d), (f).

Flame front instabilities occur during the transient conditions, which eventually decay and, as shown earlier [4], under the advanced microgravity conditions, the flame is subjected only to high-frequency oscillations. At the same time, the oscillations are so strong that the plume parts are separated from the main plume and moved downstream the flow. Figure 3 shows the temperature fields in successive time intervals, illustrating the formation, separation and removal of such a part. Such kind of flame front instabilities brings to that the flame front has the shape similar to the premixed flames under the acoustic perturbation. So it was shown the possibility to simulate such important flame characteristic as flame front instabilities under the alternating gravity conditions. The future work will be devoted to the analysis of the such kind instabilities and comparison with experimental results.

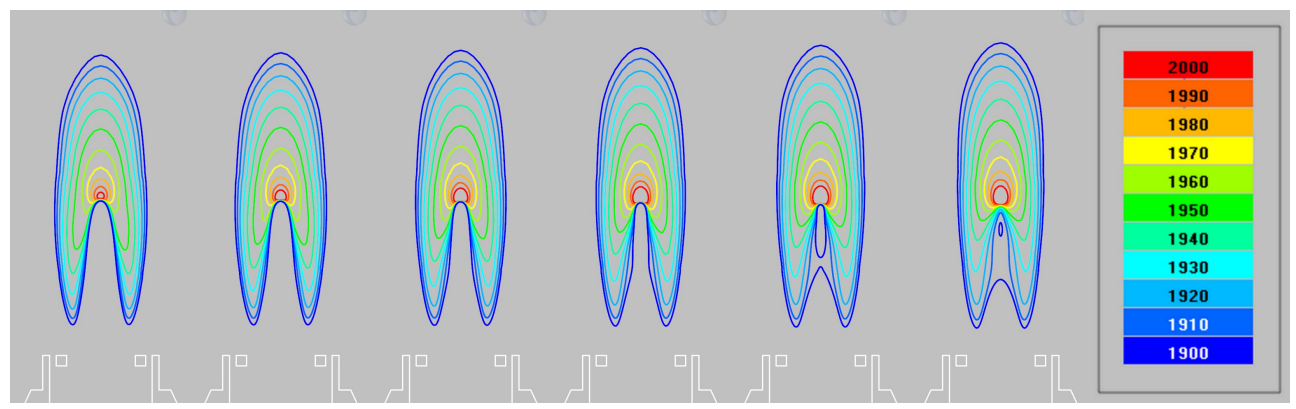

Fig. 3. Flame temperature field dynamics, time step - $0.001 \mathrm{~s}$. 
The work was supported by the Russian Science Foundation, Grant No. 18-79-00284.

\section{References}

1. H.D. Ross (Ed.), Microgravity combustion: fire in free fall (Academic press, 2001)

2. M. Dreyer, Microgravity Sci. Technol. 22, 4 (2010)

3. A.I. Krikunova, E.E. Son, High. Temp. 1 (2018)

4. A.I. Krikunova, E.E. Son, Microgravity Sci. Technol. 1 (2018)

5. A.A. Aksenov, Computer Research and Modeling 9, 5 (2017) 